\title{
"SeismoSAT" project results in connecting seismic data centres via satellite
}

\author{
Damiano Pesaresi ${ }^{1}$, Wolfgang Lenhardt ${ }^{2}$, Markus Rauch ${ }^{3}$, Mladen Živčić ${ }^{4}$, Rudolf Steiner ${ }^{2}$, Michele Bertoni $^{1}$, and \\ Heimo Delazer ${ }^{3}$ \\ ${ }^{1}$ OGS (Istituto Nazionale di Oceanografia e di Geofisica Sperimentale), Trieste, Italy \\ ${ }^{2}$ Zentralanstalt für Meteorologie und Geodynamik, Vienna, Austria \\ ${ }^{3}$ Protezione Civile della Provincia Autonoma di Bolzano, Bolzano, Italy \\ ${ }^{4}$ Agencija Republike Slovenije za Okolje, Ljubljana, Slovenia
}

Correspondence to: D. Pesaresi (dpesaresi@inogs.it)

Received: 4 December 2015 - Revised: 29 March 2016 - Accepted: 30 March 2016 - Published: 4 April 2016

\begin{abstract}
Since 2002 the OGS (Istituto Nazionale di Oceanografia e di Geofisica Sperimentale) in Udine (Italy), the Zentralanstalt für Meteorologie und Geodynamik (ZAMG) in Vienna (Austria), and the Agencija Republike Slovenije za Okolje (ARSO) in Ljubljana (Slovenia) are collecting, analysing, archiving and exchanging seismic data in real time. Up to now the data exchange between the seismic data centres relied on internet: this however was not an ideal condition for civil protection purposes, since internet reliability is poor. For this reason, in 2012 the Protezione Civile della Provincia Autonoma di Bolzano in Bolzano (Italy) joined OGS, ZAMG and ARSO in the Interreg IV Italia-Austria project "SeismoSAT" (Progetto SeismoSAT, 2014) aimed in connecting the seismic data centres in real time via satellite. As already presented in the past, the general technical schema of the project has been outlined, data bandwidths and monthly volumes required have been quantified, the common satellite provider has been selected and the hardware has been purchased and installed. Right before the end of its financial period, the SeismoSAT project proved to be successful guaranteeing data connection stability between the involved data centres during an internet outage.
\end{abstract}

\section{Introduction}

The area at the border between Slovenia, Austria and NorthEast Italy is seismically very active and was struck by many destructive earthquakes in the past. OGS, ZAMG and ARSO are running seismic networks in the area primarily for civil defence purposes. However, the single seismic networks cannot determine precisely and efficiently enough earthquakes occurring at the borders: thus since 2002 OGS, ZAMG and ARSO decided to use the same software suite Antelope (Bragato et al., 2010) as the main tool for collecting, analysing, archiving and exchanging seismic data in real time, initially in the framework of the EU Interreg IIIa Italia-Austria project "Trans-national seismological networks in the South-Eastern Alps" (Bragato et al., 2004). For many years the data exchange between the seismic data centres relied on internet: this however is not an ideal condition for civil protection purposes, since the reliability of standard internet connections is poor. Generally, internet connections can provide high bandwidth at relatively small cost, but could suffer of disruption of service in case of strong natural events like big earthquakes. Same is true for mobile GPRS/UMTS data links, where data connection reliability is even less. Satellite links, apart from the problem of antenna dislocation by strong earthquake, if provided with reliable power supply can provide more robust data connections. The SeismoSAT project makes use of satellite technology as back up for the primary internet data link between data centres. Slovenia does not belong to the Interreg Italia-Austria area of intervention: for this reason, ARSO joined the SeismoSAT project as an "associated partner", which - according to Interreg rules - cannot be funded. ARSO participation in the project is therefore limited in benefiting only indirectly from improvement in the robustness of the data exchange between the other data centres. The SeismoSAT project concluded successfully the financial period on 31 March 2015 with the last stress test of 


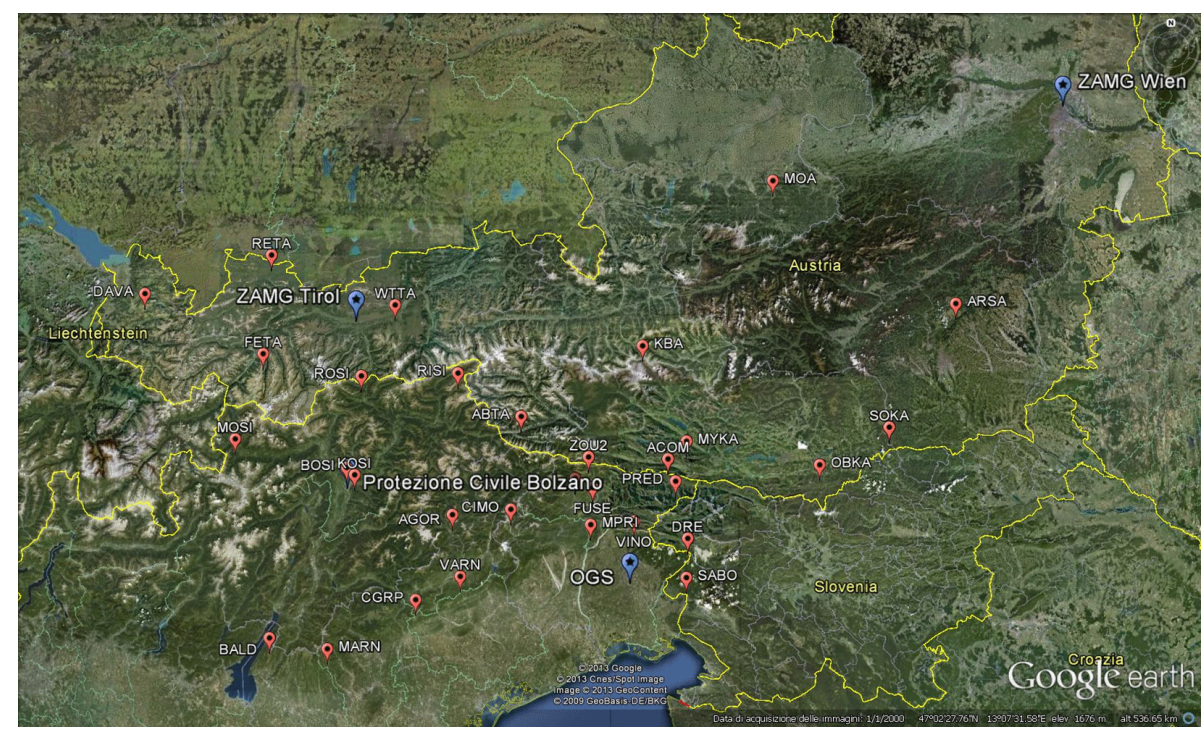

Figure 1. 2 SeismoSAT map with seismic stations in red and data centres in blue.

internet outage, showing the project capability of automatically switching to the backup satellite links. The SeismoSAT project was funded by the Interreg IV Italia-Austria (Interreg IV Italia-Austria, 2007) program based on the European Regional Development Fund (European Regional Development Fund, 2000).

\section{Project implementation}

Figure 1 shows a map of the data centres (in blue) connected by the SeismoSAT project with the corresponding seismic stations (in red). Each data centre is collecting data from the stations of its seismic network, sends a copy of it to the other two data centres and receive a copy from each other of the other two data centres. Data is primarily sent via normal internet, with the option to switch over satellite when necessary. Total upload bandwidths required are OGS $240 \mathrm{Kbit} \mathrm{s}^{-1}$, ZAMG $176 \mathrm{Kbit} \mathrm{s}^{-1}$, PCBZ $96 \mathrm{Kbit} \mathrm{s}^{-1}$, while download bandwidths required are OGS $136 \mathrm{Kbit} \mathrm{s}^{-1}$, ZAMG $168 \mathrm{Kbits}^{-1}$, PCBZ $208 \mathrm{Kbits}^{-1}$ (Pesaresi et al., 2014a).

As the satellite provider, the KA-BA satellite terminal from Sosat (Austria) has been selected. Its main characteristics are:

- max. $18432 \mathrm{kbit} \mathrm{s}^{-1}$ download, $6144 \mathrm{kbit} \mathrm{s}^{-1}$ upload

- 1 public IP address and 60 GB data volume

- Annual link availability: >99,5\%

- RTTs Satellite round trip time: $<600 \mathrm{~ms}$

- Data Interface: Lan/Ethernet

- Security: Time Division Multiple Access (TDMA)
- Modulation: 16-APSK

It proved to be the best choice in Europe for internet via satellite with data volumes like the SeismoSAT project with good technical support in the Interreg area (Pesaresi et al., 2014a).

The automatic switching between the default internet and the back-up satellite data links is done at hardware level: for this reason, all SeismoSAT partners data centres have been equipped with the Cisco 2921 router with VPN, IPSEC, ISKMP, ICMP, BGP and SSH capabilities (Pesaresi et al., 2014b). The automatic switching between the default internet and the back-up satellite data links is practically realized with the usage of several VPN tunnels between the SeismoSAT data centres: two between each couple of data centres, one via internet and one via satellite. The Cisco 2921 router, by constantly checking which of the two IP connections has the best metrics, choose automatically internet when available and switches also automatically to the back-up satellite links when internet is down.

Figure 2 shows the final schematic of the IP connections topology of the SeismoSAT project. Continuous lines are over internet, while dashed ones over satellite. The dynamic routing is realized with the Border Gateway Protocol (BGP, 2015). The SeismoSAT project had to account for different LAN configurations at the three data centres. From Fig. 2 it can be noted that the Cisco 2921 router has been installed at OGS in Udine inside the firewall, while at the ZAMG in Tyrol/Vienna is outside the firewall. Configuration at the Protezione Civile di Bolzano data centre is also different with natting. 


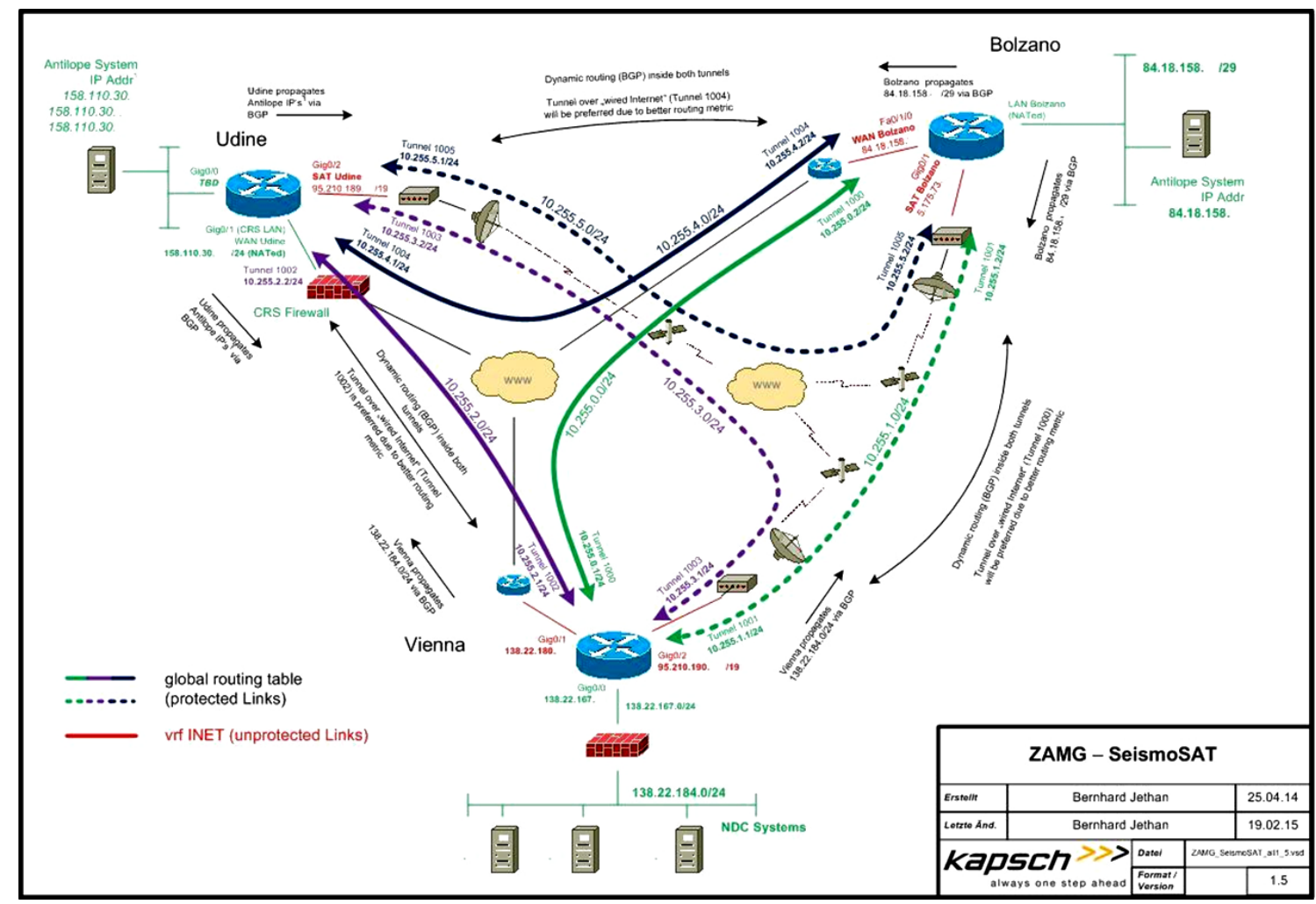

Figure 2. SeismoSAT IP connections schematic.

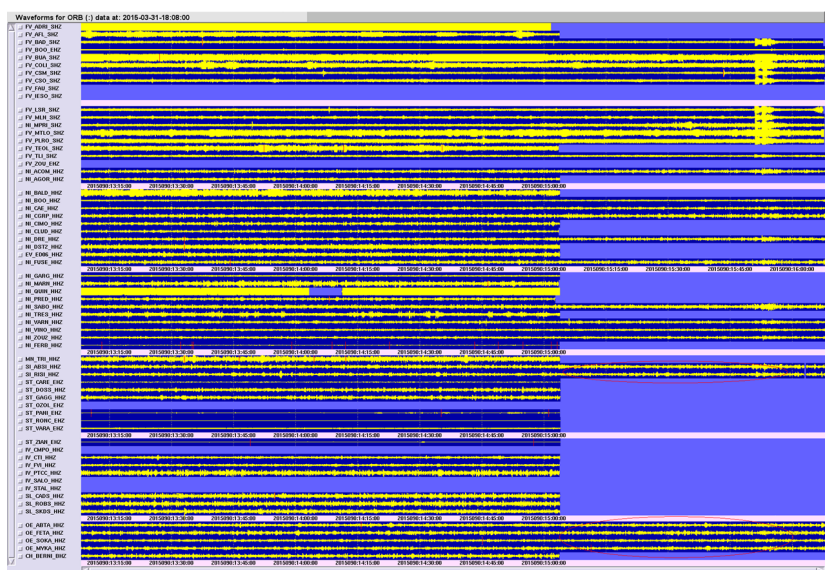

Figure 3. Stress test. With internet down, P1 ZAMG (code OE_*) and P2 PCBZ (code SI_*) seismic stations acquired at LP OGS data centre.

\section{Final test}

The final test was conducted thanks to a lucky coincidence: by chance, exactly on the last official day of the SeismoSAT project, on 31 March 2015 the OGS data centre in Udine suffered from an internet outage of several hours. Luckily the SeismoSAT infrastructure was already up and running at that time, so that seismic real time data acquisition and monitoring was not severely affected, neither at ZAMG in Tyrol/Vienna and Protezione Civile in Bolzano, nor at OGS in Udine.

Figure 3 shows a live snapshot of real time seismic waveforms acquired during the natural outage test at the OGS data centre in Udine. On the left are shown the network_station_channel labels: the OGS network code is NI, the ZAMG network code is OE and the Protezione Civile di Bolzano code is SI. From Fig. 3 it can be noted that the yellow waveform traces of the real time data acquisition at OGS in Udine stopped around 15:00 UTC for some of the stations of various seismic networks, but NOT for the SeismoSAT partners ZAMG in Tyrol/Vienna (network code OE) and Protezione Civile di Bolzano (network code SI): those yellow waveform traces appear continuous if Fig. 3 (and circled in red for reference). It must be noted that on this snapshot not all of the seismic station involved in the SeismoSAT project are showed, since it is taken form a live monitor using only a few sample stations. This proved that SeismoSAT Cisco routers correctly automatically switched over satellite data links. Similar tests were artificially conducted at each of the other data centres, thus confirming the well-functioning of the SeismoSAT project.

Table 1 shows in detail for each network_ststion_channel data latencies during the internet outage at the OGS data cen- 
Table 1. Data latency during internet outage.

\begin{tabular}{|c|c|c|c|c|c|c|c|c|c|}
\hline $\begin{array}{l}\text { Sources } \\
\text { Srcname }\end{array}$ & Thread & \#pkts & Kbytes & pktid & $\begin{array}{r}\text { Oldest } \\
\text { time }\end{array}$ & pktid & $\begin{array}{c}\text { Latest } \\
\text { time }\end{array}$ & kbps & $\begin{array}{r}\text { Average } \\
\text { latency }\end{array}$ \\
\hline EV_ED06_HHZ/SEED & 0 & 3678 & 1934.6 & 1296777 & $12: 58: 06$ & 798408 & $15: 03: 48$ & 2.1 & $1: 04 \mathrm{~h}$ \\
\hline FV_TEOL_HHZ/SEED & 0 & 2322 & 1221.4 & 1296813 & $12: 57: 55$ & 797050 & $15: 03: 40$ & 1.3 & $1: 04 \mathrm{~h}$ \\
\hline IV_BRMO_HHZ/SEED & 0 & 1835 & 965.2 & 1296795 & $12: 58: 03$ & 797055 & $15: 03: 41$ & 1.0 & $1: 04 \mathrm{~h}$ \\
\hline IV_CTI_HHZ/SEED & 0 & 1936 & 1018.3 & 1297318 & $12: 58: 05$ & 797418 & $15: 03: 44$ & 1.1 & $1: 04 \mathrm{~h}$ \\
\hline IV_FVI_HHZ/SEED & 0 & 1831 & 963.1 & 1297306 & $12: 58: 05$ & 796907 & $15: 03: 41$ & 1.0 & $1: 04 \mathrm{~h}$ \\
\hline IV_MABI_HHZ/SEED & 0 & 1787 & 940.0 & 1298796 & $12: 58: 03$ & 797024 & $15: 03: 37$ & 1.0 & $1: 04 \mathrm{~h}$ \\
\hline IV_MAGA_HHZ/SEED & 0 & 1873 & 985.2 & 1297029 & $12: 58: 05$ & 796869 & $15: 03: 41$ & 1.0 & $1: 04 \mathrm{~h}$ \\
\hline IV_PTCC_HHZ/SEED & 0 & 1831 & 963.1 & 1297336 & $12: 58: 04$ & 796691 & $15: 03: 38$ & 1.0 & $1: 04 \mathrm{~h}$ \\
\hline MN_AQU_HHZ/SEED & 0 & 3101 & 1631.1 & 1296982 & $12: 58: 02$ & 797268 & $15: 03: 31$ & 1.7 & $1: 04 \mathrm{~h}$ \\
\hline MN_BNI_HHZ/SEED & 0 & 1831 & 963.1 & 1296794 & $12: 58: 02$ & 797428 & $15: 03: 40$ & 1.0 & $1: 04 \mathrm{~h}$ \\
\hline MN_TRI_HHZ/QCDAT & 0 & 1864 & 902.2 & 1296703 & $12: 58: 02$ & 798343 & $15: 03: 45$ & 1.0 & $1: 04 \mathrm{~h}$ \\
\hline MN_TRI_HLZ/QCDAT & 0 & 1047 & 506.7 & 1296705 & $12: 58: 00$ & 797912 & $15: 03: 41$ & 0.5 & $1: 04 \mathrm{~h}$ \\
\hline MN_TUE_HHZ/SEED & 0 & 1831 & 963.1 & 1296751 & $12: 58: 01$ & 797695 & $15: 03: 41$ & 1.0 & $1: 04 \mathrm{~h}$ \\
\hline MN_VLC_HHZ/SEED & 0 & 1831 & 963.1 & 1296443 & $12: 58: 00$ & 796542 & $15: 03: 38$ & 1.0 & $1: 04 \mathrm{~h}$ \\
\hline NI_ACOM_HHZ/GENC & 44 & 11373 & 1311.9 & 1296660 & $12: 58: 06$ & 1296381 & $16: 07: 38$ & 0.9 & $2.834 \mathrm{~s}$ \\
\hline NI_AGOR_HHZ/SEED & 0 & 1832 & 963.6 & 1297119 & $12: 58: 04$ & 798410 & $15: 03: 45$ & 1.0 & $1: 04 \mathrm{~h}$ \\
\hline NI_BALD_HHZ/GENC & 0 & 7549 & 1215.3 & 1297602 & $12: 57: 59$ & 800558 & $15: 03: 47$ & 1.3 & $1: 04 \mathrm{~h}$ \\
\hline NI_BOO_HHZ/GENC & 44 & 11373 & 1295.5 & 1296593 & $12: 58: 06$ & 1296313 & $16: 07: 38$ & 0.9 & $3.122 \mathrm{~s}$ \\
\hline NI_CAE_HHZ/GENC & 44 & 11373 & 1218.9 & 1296575 & $12: 58: 06$ & 1296349 & $16: 07: 38$ & 0.9 & $3.122 \mathrm{~s}$ \\
\hline NI_CGRP_HHZ/GENC & 44 & 11373 & 1268.3 & 1296572 & $12: 58: 06$ & 1296328 & $16: 07: 38$ & 0.9 & $2.834 \mathrm{~s}$ \\
\hline NI_CIMO_HHZ/QCDAT & 0 & 1175 & 568.7 & 1297340 & $12: 58: 03$ & 797421 & $15: 03: 40$ & 0.6 & $1: 04 \mathrm{~h}$ \\
\hline NI_CLUD_HHZ/GENC & 0 & 7533 & 822.5 & 1299514 & $12: 57: 58$ & 798939 & $15: 03: 30$ & 0.9 & $1: 04 \mathrm{~h}$ \\
\hline NI_DRE_HHZ/GENC & 44 & 11373 & 1277.7 & 1296689 & $12: 58: 06$ & 1296391 & $16: 07: 38$ & 0.9 & $2.834 \mathrm{~s}$ \\
\hline NI_DST2_HHZ/GENC & 0 & 7546 & 1046.9 & 1296438 & $12: 58: 05$ & 798582 & $15: 03: 50$ & 1.1 & $1: 04 \mathrm{~h}$ \\
\hline NI_FERB_HHZ/SEED & 0 & 1831 & 963.1 & 1297023 & $12: 58: 02$ & 797626 & $15: 03: 41$ & 1.0 & $1: 04 \mathrm{~h}$ \\
\hline NI_FUSE_HHZ/GENC & 44 & 11373 & 1284.3 & 1296602 & $12: 58: 06$ & 1296372 & $16: 07: 38$ & 0.9 & $2.834 \mathrm{~s}$ \\
\hline NI_GARG_HHZ/SEED & 0 & 1831 & 963.1 & 1297113 & $12: 58: 04$ & 797700 & $15: 03: 44$ & 1.0 & $1: 04 \mathrm{~h}$ \\
\hline NI_MARN_HHZ/GENC & 0 & 7600 & 1050.7 & 1303549 & $12: 56: 50$ & 800674 & $15: 03: 29$ & 1.1 & $1: 04 \mathrm{~h}$ \\
\hline NI_MPRI_HHZ/GENC & 44 & 11373 & 1315.7 & 1296554 & $12: 58: 06$ & 1296319 & $16: 07: 38$ & 0.9 & $2.834 \mathrm{~s}$ \\
\hline NI_POLC_HHZ/GENC & 0 & 7550 & 864.3 & 1296432 & $12: 58: 05$ & 798588 & $15: 03: 50$ & 0.9 & $1: 04 \mathrm{~h}$ \\
\hline NI_PRED_HHZ/GENC & 0 & 7472 & 972.9 & 1300472 & $12: 58: 05$ & 783151 & $15: 02: 36$ & 1.0 & $1: 05 \mathrm{~h}$ \\
\hline NI_PURA_HHZ/GENC & 0 & 7572 & 1118.7 & 1296770 & $12: 57: 56$ & 797138 & $15: 03: 43$ & 1.2 & $1: 04 \mathrm{~h}$ \\
\hline NI_QUIN_HHZ/SEED & 0 & 2861 & 1504.9 & 1297103 & $12: 58: 05$ & 798236 & $15: 03: 47$ & 1.6 & $1: 04 \mathrm{~h}$ \\
\hline NI_SABO_HHZ/GENC & 44 & 11373 & 1277.6 & 1296563 & $12: 58: 06$ & 1296337 & $16: 07: 38$ & 0.9 & $2.834 \mathrm{~s}$ \\
\hline NI_TRES_HHZ/SEED & 0 & 2304 & 1211.9 & 1296515 & $12: 58: 02$ & 798436 & $15: 03: 47$ & 1.3 & $1: 04 \mathrm{~h}$ \\
\hline NI_VARN_HHZ/GENC & 44 & 11373 & 1381.8 & 1296651 & $12: 58: 06$ & 1296346 & $16: 07: 38$ & 1.0 & $2.834 \mathrm{~s}$ \\
\hline NI_VINO_HHZ/QCDAT & 36 & 2670 & 1292.3 & 1296700 & $12: 58: 02$ & 1296175 & $16: 07: 33$ & 0.9 & $8.535 \mathrm{~s}$ \\
\hline NI_ZOU2_HHZ/GENC & 44 & 11373 & 1126.7 & 1296584 & $12: 58: 06$ & 1296358 & $16: 07: 38$ & 0.8 & $2.834 \mathrm{~s}$ \\
\hline OE_ABTA_HHZ/GENC & 54 & 11377 & 1230.6 & 1296462 & 12:58:01 & 1296406 & $16: 07: 37$ & 0.9 & $3.834 \mathrm{~s}$ \\
\hline OE_ARSA_HHZ/QCDAT & 54 & 1297 & 627.7 & 1297286 & $12: 58: 01$ & 1295510 & $16: 07: 23$ & 0.4 & $18.182 \mathrm{~s}$ \\
\hline OE_DAVA_HHZ/QCDAT & 54 & 1341 & 649.0 & 1296984 & $12: 57: 59$ & 1296040 & $16: 07: 28$ & 0.5 & $13.765 \mathrm{~s}$ \\
\hline OE_FETA_HHZ/GENC & 54 & 11376 & 1125.7 & 1296459 & $12: 58: 01$ & 1296285 & $16: 07: 36$ & 0.8 & $4.834 \mathrm{~s}$ \\
\hline OE_KBA_HHZ/QCDAT & 54 & 1279 & 619.0 & 1297267 & $12: 58: 00$ & 1295894 & $16: 07: 25$ & 0.4 & $16.691 \mathrm{~s}$ \\
\hline OE_KBA_HLZ/QCDAT & 54 & 1262 & 610.8 & 1297637 & $12: 58: 02$ & 1296026 & $16: 07: 27$ & 0.4 & $14.579 \mathrm{~s}$ \\
\hline OE_MOA_HHZ/QCDAT & 54 & 1430 & 692.1 & 1297120 & $12: 58: 00$ & 1295586 & $16: 07: 24$ & 0.5 & $16.904 \mathrm{~s}$ \\
\hline OE_MYKA_HHZ/GENC & 54 & 11377 & 1179.6 & 1296453 & 12:58:01 & 1296403 & $16: 07: 37$ & 0.8 & $3.834 \mathrm{~s}$ \\
\hline OE_SOKA_HHZ/GENC & 54 & 11377 & 1171.8 & 1296456 & $12: 58: 01$ & 1296409 & $16: 07: 37$ & 0.8 & $3.834 \mathrm{~s}$ \\
\hline OE_WTTA_HHZ/GENC & 54 & 11376 & 1415.9 & 1296527 & $12: 58: 02$ & 1296412 & $16: 07: 37$ & 1.0 & $3.834 \mathrm{~s}$ \\
\hline SI_ABSI_HHZ/GENC & 185 & 11345 & 1234.6 & 1296665 & $12: 58: 05$ & 1296299 & $16: 07: 37$ & 0.9 & $3.834 \mathrm{~s}$ \\
\hline SI_BOSI_HHZ/GENC & 185 & 11346 & 2199.8 & 1296636 & $12: 58: 05$ & 1296416 & $16: 07: 37$ & 1.5 & $3.834 \mathrm{~s}$ \\
\hline SI_KOSI_HHZ/GENC & 185 & 11345 & 1276.7 & 1296719 & $20: 43: 10$ & 1296278 & $23: 52: 41$ & 0.9 & 15 years \\
\hline SI_LUSI_HHZ/GENC & 185 & 11345 & 1279.8 & 1296671 & $12: 58: 05$ & 1296302 & $16: 07: 37$ & 0.9 & $3.834 \mathrm{~s}$ \\
\hline SI_MOSI_HHZ/GENC & 185 & 11346 & 1418.0 & 1296465 & $12: 58: 05$ & 1296419 & $16: 07: 37$ & 1.0 & $3.834 \mathrm{~s}$ \\
\hline SI_RISI_HHZ/GENC & 185 & 11346 & 1248.9 & 1296447 & $12: 58: 04$ & 1296305 & $16: 07: 37$ & 0.9 & $3.834 \mathrm{~s}$ \\
\hline
\end{tabular}


Table 1. Continued.

\begin{tabular}{lrrrrrrrr}
\hline Sources & Thread & \#pkts & Kbytes & pktid & $\begin{array}{r}\text { Oldest } \\
\text { time }\end{array}$ & pktid & $\begin{array}{r}\text { Latest } \\
\text { time }\end{array}$ & $\begin{array}{r}\text { Average } \\
\text { kbps }\end{array}$ \\
latency
\end{tabular}

tre: it can be noted that latencies for SeismoSAT partner networks were normal (within seconds), while other networks suffered more than one hour of outage.

\section{Conclusions}

As shown in Fig. 3, the Interreg IV Italia-Austria SeismoSAT project proved to be successful before the financial closing date of 31 March 2015: the backup satellite links have been operational ever since. The Civil Defence of the Friuli Venezia Giulia region expressed its interest in extending the SeismoSAT project to its headquarters in Palmanova and is considering to guarantee the future sustainability of the project.

Acknowledgements. The authors wish to thank the Interreg IV Italia-Austria (2014) Authority for the financial support on the ERDF (2014) Fund and all the colleagues of OGS, ZAMG, Protezione Civile della Provincia Autonoma di Bolzano and ARSO not explicitly mentioned here for their support to the SeismoSAT project.

Edited by: H. Pedersen

Reviewed by: two anonymous referees

\section{References}

Border Gateway Protocol (BGP): https://en.wikipedia.org/wiki/ Border_Gateway_Protocol, last access: 4 December 2015.

Bragato, P. L., Costa, G., Fitzko, F., Horn, N., Priolo, E., Kobal, M., Suhadolc, P., and Živčić, M.: The INTERREG IIIA Project: "Trans-National Seismological Networks in the South-eastern Alps", ESC XXIX General Assembly, Potsdam, Germany, 1317 September 2004, 2004.

Bragato, P. L., Costa, G., Horn, N., Pahor, J., Pesaresi, D., Lenhardt, W., and Živčić, M.: The usage of Antelope for acquiring end exchanging data in South-Eastern Alps: present configuration and future perspectives, ESC XXXII General Assembly, Montpellier, France, 6-10 September 2010, 2010.

European Regional Development Fund (ERDF): http://ec.europa. eu/regional_policy/index.cfm/en/funding/erdf/ (last access: 22 October 2015), 2000.

Interreg IV Italia-Austria: http://www.interreg.net/ (last access: 22 October 2015), 2007.

Pesaresi, D., Lenhardt, W., Rauch, M., Živcic, M., Steiner, R., Fabris, P., and Bertoni, M.: The Interreg IV Italia-Austria "SeismoSAT" project: connecting seismic data centers via satellite, Adv. Geosci., 36, 57-60, doi:10.5194/adgeo-36-57-2014, 2014a.

Pesaresi, D., Lenhardt, W., Rauch, M., Živčić, M., Steiner, R., and Bertoni, M.: "SeismoSAT" project state of the art: connecting seismic data centres via satellite, EGU General Assembly 2014, Vienna, Austria, 27 April-2 May 2014, doi:10.13140/RG.2.1.1382.6089, 2014b.

Progetto SeismoSAT: http://www.crs.inogs.it/seismosat/ (last access: 22 October 2015), 2014. 